\title{
The Long-term Outcomes of Hepatic Resection Between Single Tumor and Multiple Tumors of HCC Meeting UCSF Criteria
}

\section{Jinli Zheng}

west china hospital

Wei Xie

west china hospital

Yang Huang

west china hospital

Xingyu Pu

West China Hospital

Li Jiang ( $\nabla$ jianglihuaxi@163.com )

Sichuan University West China Hospital

Research article

Keywords: Hepatocellular carcinoma, UCSF, Multiple Tumors, Single Tumor, Hepatic Resection (HR)

Posted Date: January 18th, 2021

DOI: https://doi.org/10.21203/rs.3.rs-146720/v1

License: (c) (1) This work is licensed under a Creative Commons Attribution 4.0 International License. Read Full License 


\section{Abstract}

Object: Searching the hepatectomy of hepatocellular carcinoma (HCC) within UCSF is limited. The study aims to compare the long-term surgical outcomes between single and multiple tumors to find an effective and safe treatment for the patients within UCSF criteria.

Methods: We have enrolled 1006 patients meeting UCSF criteria, including 744 patients with single tumor and 262 patients with multiple tumors undergoing hepatic resection (HR). We divided the patients into two groups: single tumor group and multiple tumor group. By comparing the long-term outcomes between these two groups and analyzing the risk factors by the log-rank test and cox proportional hazards model in two groups.

Results: The 1-, 3-, and 5-year OS rates in the single tumor group were significantly higher than the multiple tumors group $(96.2 \%, 65.3 \%$ and $40.1 \%$ versus $93.1 \%, 47.6 \%$ and $22.5 \%$, respectively, $\mathrm{p}<0.001)$. The 1-, 3- and 5-year RFS rates were $91.4 \%, 46.2 \%$, and $24.6 \%$ in the single group and $90.8 \%, 31.1 \%$ and $13.0 \%$, respectively $(p<0.001)$. And the anatomic resection and MVI were the independent risk factors for single tumor, but it didn't work for multiple tumors. The independent risk factors of multiple tumors are the diameter more than $2 \mathrm{~cm}$ of the maximum tumor and the tumors located in different segments.

Conclusion: The surgical resection will obtain a better outcomes for the HCC with single tumor meeting UCSF criteria than the multiple tumors. And the anatomic resection should be applied for the patients with single tumor, if possible.

\section{Introduction}

Hepatocellular carcinoma (HCC) is the sixth most malignant tumor in the word and causes about 600,000 patients deaths annually[1], ranking the third place of cancer-related death. The treatments of HCC are including: liver transplantation (LT), hepatic resection (HR), radiofrequency ablation (RFA), transarterial chemoembolization (TACE) and so on. Although the LT is the optimal treatment for the patients with HCC in early stage [2], especially for the patients meeting Milan criteria or University of California San Franciso (UCSF) criteria, the shortage of organs limit its feasibility [3], meaning that not every patient with HCC can receive the LT timely. Therefore, HR is still as a worldwide treatment for $\mathrm{HCC}$ and the long-term outcomes is comparable to LT for HCC [4].

UCSF criteria is one of the criterion for LT and the patients who met UCSF criteria also can receive surgical resection. However, the outcomes of HR for the patients within UCSF is limited. Terence C et al [4] reported the patients meeting UCSF criteria undergoing HR can achieve a comparable long-term outcomes comparing with Milan criteria. But it didn't analyze the different types of the tumors ( single and multiple) and the sample is small. As the previous studies mentioned that the multiple tumors are an important factor to predict the patients with microvascular invasion (MVI) resulting a poor prognosis $[5,6$, $7,8]$. On the other hand, the studies searching the treatment of mutifocal tumors within Milan criteria have been reported in several studies $[9,10,11]$, but these studies didn't analyze the long-term outcomes 
in single tumor. To the best of our knowledge, the long-term outcomes of single and multiple tumors within exceptible is unclear, especially for the tumors within UCSF, which needs a further investigation.

As to the UCSF criteria contains the Milan criteria. The more patients would get benefits if we can figure out the risk factors and which methods to resection tumors is more suitable for the patients within UCSF criteria. The study aims to retrospect the patients who met UCSF criteria undergoing HR, and to compare the long-term outcomes of different tumor types, and to assist the surgeons to find an effective and safe treatment for the different tumor types within UCSF criteria.

\section{Patients And Methods}

This study was approved by the West China Hospital Ethics Committee and was performed in accordance with the ethical guidelines of the Declaration of Helsinki.

\section{Patients}

We retrospected the patients with HCC in our center, department of liver surgery, liver transplantation center, West China Hospital of Sichuan University, from January 2008 to January 2018. The patients were enrolled as followings: 1) Age $>18$ years old, including male and female; 2) Meeting UCSF criteria; 3 ) Without other tumors therapy history, especially the recurrence of HCC; 4) Without other fatal disease, such as heart disease and respiratory insufficiency; 5) didn't receive the treatment of LT. If the patient could not meet one of the followings, he would be excluded.

\section{Methods}

We divided the patients into two groups according to the numbers of tumors (sing tumor group and multiple tumors group). And compared the long-term outcomes between the groups and analyzed the independent risk factors in these two groups. Then we analyzed the independent risk factors in sing tumor group and multiple tumors group, respectively.

\section{Definitions and Diagnostic Criteria}

The UCSF criteria is defined as a single tumor with the diameter $\leq 6.5 \mathrm{~cm}$ or the maximum diameter of 2-3 tumors $\leq 4.5 \mathrm{~cm}$ in patients without extrahepatic manifestations and vascular invasion from imaging[12].

The patients with HCC were diagnosed by a histopathological examination after resection.

Anatomical resection (AR) is followed to the Glisson's Pedicles to resect the liver lesion. And local resection is also called non-anatomical resection (NAR), defined as the resection is focused on resect the tumor completely and left the volume of liver enough possibly to avoid the post-operative liver failure, without concerning about the Glisson's Pedicles.[13,14]

\section{Preoperative evaluation}


All patients were informed about the treatments including LT, HR, RFA and TACE. The liver function, blood test, coagulation function and the imaging examination were under reviewed by the surgeons with more than 5-year experience in hepatectomy.

\section{The procedures in HR}

Surgical procedures in HR are similar to the previous studies [15,16]. Briefly, the surgeons opened the abdomen standing on the right side of the patient with a right subcostal incision, and extending the incision along the mid-line of the sternum. Every patient undergoing HR would have a Intraoperative ultrasonography to confirm the tumors boundaries and to identify the relationship of the portal vein, hepatic artery and hepatic vein that to give a guide line to hepatectomy; and to find whether there existed another lesion that we can not judge from imaging by naked eye.

\section{Follow-up and treatment of recurrence}

All patients consulted a doctor after operation in the outpatient clinic, routinely. A-fetoprotein (AFP) and hepatitis $B$ virus deoxyribonucleic acid (HBV DNA) measures and abdominal ultrasonography were performed every 3 months. Contrast enhanced CT scans were performed every 6 months. When the recurrence was difficult to diagnose, contrast enhanced MRI or ultrasonography was performed. The patients with recurrence were treated as the following: re-resection, RFA, LT, TACE and chemotherapy.

\section{Statistical analysis}

SPSS 22.0 statistical software (SPSS Inc., Chicago, IL, USA) was used to analyze the relevant data. The categorical data are presented as the number (percent) and were compared using Pearson chi-square or Fisher's exact test. The continuous variables are expressed as the mean value $\pm S D$ and were analyzed by the $t$ test. The overall survival (OS) rates and recurrence-free survival (RFS) rates were estimated by the Kaplan-Meier method, and the differences between groups were determined by the log-rank test. A Cox proportional hazards model was used to test potential predictors of survival. A 2-tailed $\mathrm{P}<0.05$ was considered statistically significant.

\section{Results}

\subsection{The baseline of the patients}

We have enrolled 1006 patients with HCC meeting UCSF criteria undergoing HR, including 744 patients with single tumor and 262 patients with multiple tumors (146 patients in same segment (SS) and 116 patients in different segments (DS)). Among the patients about 859 are male and 147 are female. The Table 1 shows the characteristic of patients in the two types of the tumors. The significant difference between the groups is just blood platelet (PLT) count ( $111.39 \pm 53.14$ versus $120.41 \pm 56.59, p=0.024$ ) . But the levels of PLT between the groups is in the normal range. And there were no deaths within 30 days after operation. 
Table 1

The characteristic of the patients

\begin{tabular}{|llll|}
\hline Variable & Multiple & Single & P value \\
\hline Sex (male, ratio) & $226(86.26 \%)$ & $633(85.08 \%)$ & 0.642 \\
\hline Age (years) & $51.93 \pm 12.15$ & $52.29 \pm 11.93$ & 0.677 \\
\hline HGB (g/L) & $141.31 \pm 18.37$ & $139.43 \pm 18.00$ & 0.225 \\
\hline WBC $\left(\times 10^{9} / \mathrm{L}\right)$ & $5.37 \pm 2.24$ & $6.12 \pm 2.74$ & 0.222 \\
\hline PLT (× $\left.10^{9} / \mathrm{L}\right)$ & $111.39 \pm 53.14$ & $120.41 \pm 56.59$ & 0.024 \\
\hline CHB (positive, ratio) & $233(88.93 \%)$ & $635(85.35 \%)$ & 0.147 \\
\hline Serum AFP ( $\geq 400$ ng/mL, ratio) & $97(37.02 \%)$ & $248(33.33 \%)$ & 0.279 \\
\hline TB ( $\mu$ mol/L) & $15.81 \pm 6.88$ & $17.69 \pm 7.37$ & 0.200 \\
\hline AST ( IU/L) & $50.32 \pm 61.15$ & $54.91 \pm 97.35$ & 0.475 \\
\hline ALT ( IU/L) & $58.82 \pm 71.63$ & $65.47 \pm 80.37$ & 0.424 \\
\hline ALB (g/L) & $41.54 \pm 5.44$ & $41.27 \pm 5.70$ & 0.495 \\
\hline PT (s) & $12.13 \pm 1.38$ & $12.00 \pm 1.32$ & 0.196 \\
\hline MVI (positive, ratio) & $58(22.14 \%)$ & $145(19.49 \%)$ & 0.358 \\
\hline Anatomic resection (yes, ratio) & $150(57.25 \%)$ & $441(59.27 \%)$ & 0.567 \\
\hline Different segments (yes, ratio) & $116(44.27 \%)$ & - & - \\
\hline $\begin{array}{l}\text { Abbreviation: HGB: hemoglobin; WBC: white blood cell; PLT: platelet; CHB: chronic hepatitis B virus; } \\
\text { AFP: A-fetoprotein; TB: total bilirubin; AST: aspartate aminotransferase; ALT: ananine transaminase; }\end{array}$ & \\
\hline ALB: albumin; PT: prothrombin time; MVl: microvascular invasion. & & \\
\hline
\end{tabular}

\subsection{Overall Survival}

The median survival time of single and multiple tumors are 49.9 (range, 2.9-88.1) months and 33.4 (range, 2.8-84.2) months, respectively. During the follow-up period, $402(54.03 \%)$ patients have passed away in the single tumor group and 179 (68.32\%) patients have died in the multiple tumors group. The 1-, $3-$, and 5-year OS rates in the single tumor group were significantly higher than the multiple tumors group $(96.2 \%, 65.3 \%$ and $40.1 \%$ versus $93.1 \%, 47.6 \%$ and $22.5 \%$, respectively, $\mathrm{p}<0.001$; Fig $1-\mathrm{A})$. There were 513 (68.95\%) patients with tumor recurrence in the single tumor group and $211(80.53 \%)$ patients in the multiple tumors group. The 1-, 3- and 5-year RFS rates were $91.4 \%, 46.2 \%$, and $24.6 \%$ in the single group and $90.8 \%, 31.1 \%$ and $13.0 \%$, respectively ( $p<0.001$, Fig. $1-B$ ). According to univariate and multivariate analysis ( Table 2 and Table 3), we found that the independent prognostic factors were not only the number of tumors, but also including anatomic resection and MVI (Fig.1-C, 1-D, 1-E,1-F). 
Table 2

Univariate analysis of the prognostic factors for survival and recurrence

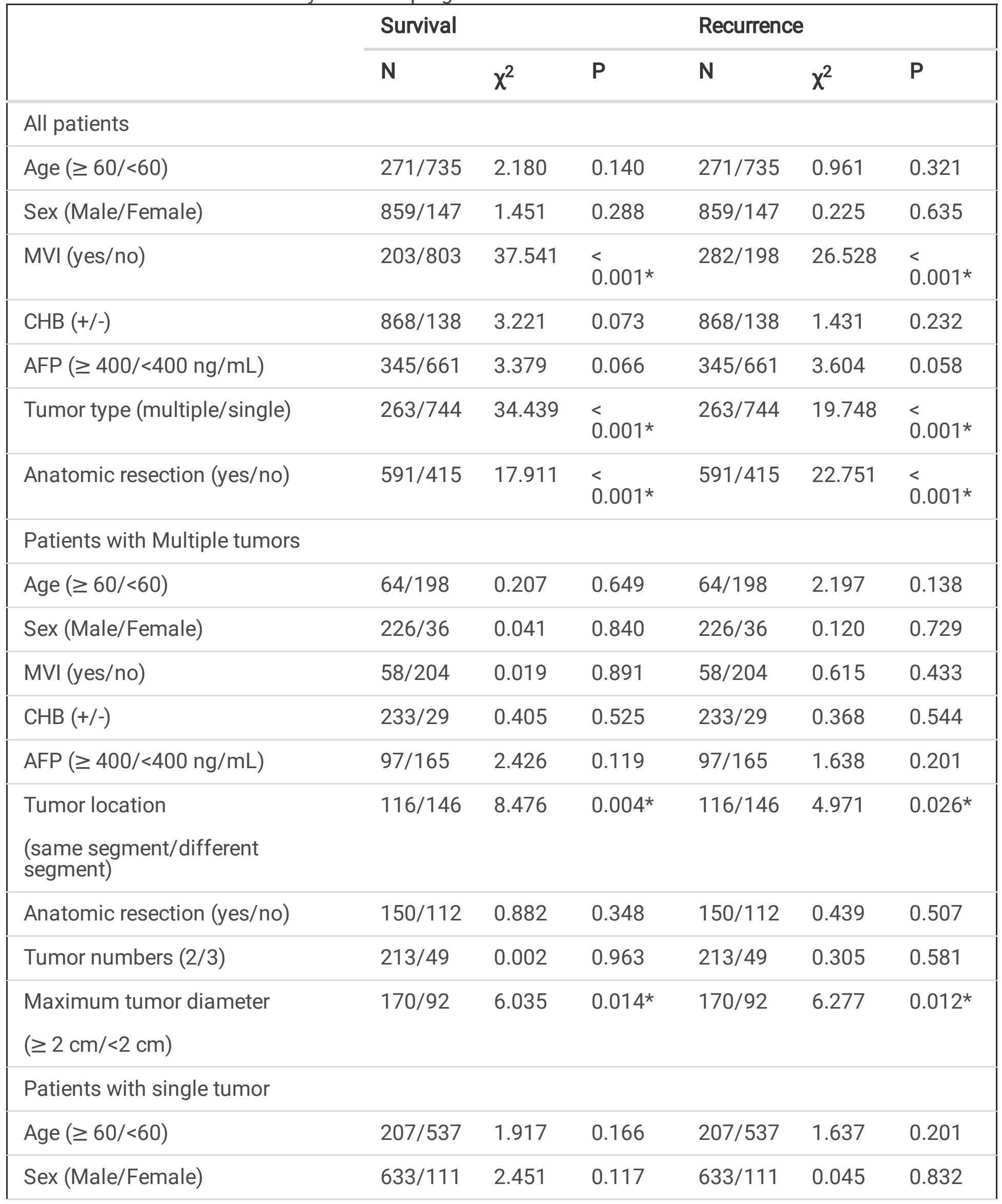




\begin{tabular}{|lllllll|}
\hline & \multicolumn{5}{c}{ Survival } & \multicolumn{5}{c|}{ Recurrence } \\
\cline { 2 - 7 } & N & $\chi^{2}$ & P & N & $\chi^{2}$ & P \\
\hline MIV (yes/no) & $145 / 599$ & 53.134 & $<$ & $145 / 599$ & 35.567 & $<$ \\
\hline CHB $(+/-)$ & & & $0.001^{*}$ & & & $0.001^{*}$ \\
\hline AFP $(\geq 400 /<400 \mathrm{ng} / \mathrm{mL})$ & $635 / 109$ & 4.363 & $0.037^{*}$ & $635 / 109$ & 2.691 & 0.101 \\
\hline Anatomic resection $(\mathrm{yes} / \mathrm{no})$ & $441 / 303$ & 7.823 & $0.005^{\star}$ & $441 / 303$ & 14.503 & $<$ \\
\hline Tumor diameter & & & & & & $0.001^{*}$ \\
$(\geq 4 \mathrm{~cm} /<4 \mathrm{~cm})$ & $417 / 327$ & 1.829 & 0.176 & $417 / 327$ & 0.876 & 0.349 \\
\hline
\end{tabular}

Table 3

Multivariate analysis of the prognostic factors for survival

\begin{tabular}{|lccl|}
\hline Variable & HR & P value & $95 \% \mathrm{Cl}$ \\
\cline { 3 - 4 } & & & Down Up \\
\hline All patients & & & \\
\hline Tumor type (multiple) & 1.553 & $<0.001^{*}$ & 1.2911 .869 \\
\hline MVI (Yes) & 1.756 & $<0.001^{*}$ & 1.4442 .136 \\
\hline Anatomic resection (Yes) & 0.802 & $0.011^{*}$ & 0.6780 .950 \\
\hline Patients with Multiple tumors & & & \\
\hline Tumor location (same segment) & 0.677 & $0.012^{*}$ & 0.4980 .919 \\
\hline Maximum tumor diameter ( $\geq 2 \mathrm{~cm})$ & 1.381 & $0.049^{*}$ & 1.0011 .924 \\
\hline Patients with single tumor & & & \\
\hline Anatomic resection (Yes) & 0.735 & $0.002^{*}$ & 0.6030 .895 \\
\hline MVI (Yes) & 2.353 & $<0.001 *$ & 1.8652 .968 \\
\hline Abbreviation: HR: hazard ratio, Cl: confidence interval & \\
\hline
\end{tabular}

\subsection{The survival for the patients with single tumor}

As showing in Table 2 and Table 3 , the overall survival rates of the patients with single tumor is closely related to the anatomic resection and MVI (Table 2 and Table 3). And the resection of anatomic resection 
may act as a protective factor and the patients with MVI-positive are higher risk to recurrence, affecting the patients' survival (Fig. 2-A, 2-B, 2-C, 2-D).

\subsection{The survival for the patients with multiple tumors}

The independent risk factors of the multiple tumors are just included the diameter more than $2 \mathrm{~cm}$ of the maximum tumor and the tumors located in different segments ( Table 2 and Table 3 ). The MVI and anatomic resection may not play a role in patients with multiple tumors, which is different with the single tumor. The OS rates and RFS rates are showed in Fig 3-A, 3-B, 3-C and 3-D, respectively.

\section{Discussion}

In the current study, we compared the surgical outcomes meeting the UCSF criteria by retrospecting a large cohort of HCC patients. We found that the patients with single tumor had significantly better OS rates and RFS rates after HR than the patients with multiple tumors (Fig 1-A and 1-B). According to Kaplan-Meier analysis and multivariate Cox modeling analysis, the tumor numbers (single tumor, Fig 1-A and 1-B), the methods of HR (anatomic resection, Fig 1-C and 1-D) and MVI (negative, Fig 1-E and 1-F) are independent better prognosis factors in the patients (table 2 and table 3 ). Previous studies $[17,18]$ have reported that the number of nodules was an independent risk factor in recurrence, which is mainly caused by metastatic recurrence from the main tumor via the portal system [19]. And the MVI playing an independent risk factor of recurrence and survival has been proved by previous studies $[5,6,7,8]$.

In our study, we analyzed the independent risk factors in single tumor and multiple tumors, respectively. We found that the difference of MVI and anatomic resection were no significance in the patients with multiple tumors, but the tumors located in different segments and the maximum tumors' diameter more than $2 \mathrm{~cm}$ had a great influence on the prognosis of these patients (Fig 3-A, 3-B, 3-C and 3-D), which may be explained by Adachi E et al reported [19] and similar to the previous findings [10]. The multiple tumors may mean that the tumor has invaded into different sites of portal vein system, especially for the tumors located in different segments. The tumors located in different hepatic areas, meaning the tumors were more possible intrahepatic spread for the tumors in different branch of portal vein resulting the recurrence higher and poor outcomes. And the anatomic resection couldn't resect the potential invaded segments so that the MVI may lose its function in prognosis in these patients. Generally, the patients with multiple tumors may be metastatic by the portal system, though the surgical margin was informed the MVInegative from histopathological examination. Therefore, the patients with multiple tumors were highly risk of recurrence, which is the major risk factor affecting the survival $[20,21]$. This can be a reason for the anatomic resection and $\mathrm{MVI}$ were no significance in these patients, and explain the bad prognosis of multiple tumors compared with single tumor. But the OS and RFS rates of the anatomic resection are higher than local resection (Fig 1-C and 1-D).

On the other hand, the anatomic resection and MVI were very important for the patients with single tumor (Fig 2-A, 2-B, 2-C and 2-D). In previous studies [22,23] have shown that anatomic resection has better surgical outcomes than the nonanatomic resection. So the patients with single tumor may have a better 
outcomes in anatomic resection than the local resection (Fig 2-A and 2-B). MVI was an important risk factor in recurrence $[5,6,7,8]$, though it didn't make sense in the multiple tumors in this study. In our study, the patients with MVI-negative in single tumor group has a better outcomes than MVI-positive (Fig 2-C and 2-D). MVI is correlated with the tumors' diameter, and the previous findings pointed out the tumors' diameter more than $4 \mathrm{~cm}$ is likely with MVI $[24,25]$, but the tumors' diameter more than $4 \mathrm{~cm}$ has no significant difference in survival and recurrence in our study. On the other hand, a survey was carried by Yan-Yan Wang et al shown that the tumors' diameter more than $7 \mathrm{~cm}$ can be more accurate to predict the prognosis of HCC patients [26]. However, the relationship of MVI and tumors' diameter didn't analyze in the current study, then it needs a further research in the future, because the patients with MVI-positive are 2.35 folds to die within 5 years after operation compared to the MVI-negative (Table 3 ).

The number of tumors is an independent risk factor affecting the survival rate [17,18], but it didn't analyze the role of tumors' diameter in the previous studies. In multiple tumors group, the maximum tumors' diameter more than $2 \mathrm{~cm}$ is an independent risk factor and it is 1.38 folds to die within 5 years after operation (table 3), which may present a message that the faster the tumor grows, the more malignant it becomes and the more aggressive behaviors it has. That is maybe a explanation for the tumors' diameter is a risk factor for the patients. We haven't analyzed the maximum tumors' diameter in stratification by $1 \mathrm{~cm}$ and $3 \mathrm{~cm}$, because the patient with tumors' diameter smaller than $1 \mathrm{~cm}$ is 11 , and the patient with tumors' diameter lager than $3 \mathrm{~cm}$ is 15 . But we analyzed the diameter more than $2 \mathrm{~cm}$ and found it was a risk factor affecting the patients survival, which may need a further research.

The high levels of AFP has been proved having more aggressive behaviors in previous findings [27,28], especially for the levels of AFP higher than $1000 \mathrm{ng} / \mathrm{mL}$. On the other hand, the levels of AFP higher than $400 \mathrm{ng} / \mathrm{mL}$ is a risk factor in huge tumors [29,30]. In the current study, the levels of AFP ( $\geq 400 \mathrm{ng} / \mathrm{mL})$ has no significant difference. The main reason is that we hasn't compared the AFP in different levels, separately. The hepatic B virus (HBV) infection is the mainly reason for patients with HCC in China, and the patients with HBV are more likely to develop into cirrhosis, which is also a risk factor in recurrence and bad prognosis $[31,32,33]$. The patients with HBV were no significant influence in both groups. This may be explained as we will take antiviral therapy to control the viral replication and to slow down the progress of cirrhosis. And the other risk factor is gene [34], which has been reported that p53- and AFPpositive were predictors for poor prognosis of $\mathrm{HCC}$ after hepatic resection. It is a shortcoming that we didn't do genetic testing between these patients.

The study also has its other limitations. Firstly, it was a retrospective study involving the experience of a single centre with small sample. Secondly, we haven't compared the outcomes with liver transplantation, Milan criteria and radiofrequency ablation for the patients. Finally, the retrospective study has the biases, especially for the long-term outcomes follow-up. However, to the best of our knowledge, this study is the first search focusing on the outcomes of HR in the patients meeting UCSF criteria, analyzing the single and multiple tumors' outcomes. Based on our study findings, the OS rates and RFS rates of HR are higher in the single tumor group than in the multiple tumors group, and the difference between the tumor located in the different segments and in the same segment is significant for the multiple tumors group. The 
anatomic resection and MIV may have no sense in the multiple tumor group, but they played an important role in the single tumor group.

\section{Conclusion}

The surgical resection will obtain a better outcomes for the HCC with single tumor meeting UCSF criteria than the multiple tumors. The anatomic resection should be applied for the patients with single tumor, for which could be a protective factor to avoid recurrence and do a favor for the long-term outcomes.

\section{Abbreviation}

HCC: hepatocellular carcinoma, UCSF: University of California San Franciso, LT: liver transplantation, HR: hepatic resection, RFA: radiofrequency ablation, TACE: transarterial chemoembolization, OS: overall survival, RFS; recurrence-free survival, HGB囚hemoglobin; WBC: white blood cell; PLT: platelet; CHB: chronic hepatitis B virus; AFP: A-fetoprotein; TB: total bilirubin; AST: aspartate aminotransferase; ALT: ananine transaminase; ALB: albumin; PT: prothrombin time; MVI: microvascular invasion. DS: different segments, SS: same segment, N: No, Y: Yes.

\section{Declarations}

\section{Consent for publication}

Not applicable.

\section{Availability of data and materials}

The data sets used during the current study are available from the corresponding author on reasonable request.

\section{Competing interests}

The authors declare that they have no competing interests.

\section{Funding}

This study was supported by grants from the National Sciences (2012ZX10002-016) and Technology Major Project of China(2012ZX10002-017), provided by JY Yang, providing to collect the data. And the National Natural Science Foundation of China (81400636), Sichuan Province Key Research and Development Project (2019YFS0203), and the Key Project of Clinical Research Incubation in West China Hospital of Sichuan University (2020HXFH028), provided by L Jiang, to supported the study designing, data analysis and interpretation, writting the manuscript and scientific language editing, respectively.

\section{Author's contribution}


Author Contributions:Study conception and design: LJ; Acquisition of data: JLZ, WX and YH; Collected and copied imaging picture: YH and JLZ; Analysis and interpretation of data: LJ and JLZ; Drafting of manuscript: JLZ and WX; Critical revision: LJ; XYP and JLZ contributed in statistical analysis. All authors have read and approved the manuscript.

\section{Acknowledgments}

Thanks for the www. enago. cn provided scientific language editing service. And the funding supported by JY Yang and LJ.

\section{References}

1. Zhao C, Nguyen $\mathrm{MH}$. Hepatocellular carcinoma screening and surveillance: practice guidelines and real-life practice. J Clin Gastroenterol. 2016;50:120-33.

2. Mazzaferro V, Regalia E, Doci R, Andreola S, Pulvirenti A,Bozzetti F, et al. Liver transplantation for the treatment of small hepatocellular carcinomas in patients with cirrhosis. N Engl J Med 1996;334:693699.

3. Pruett TL. The allocation of livers for transplantation: a problem of Titanic consideration. Hepatology. 2002;35:960-963.

4. Chua TC, Saxena A, Chu F, Morris DL. Hepatic Resection for Transplantable Hepatocellular Carcinoma for Patients Within Milan and UCSF Criteria. American Journal of Clinical Oncology. 2012,4(2):141-145

5. Dong ZR, Zhang PF, Wang CH, Zhang C, Cai JB, Shi GM et al. Postoperative adjuvant transcatheter arterial chemoembolization for resectable multiple hepatocellular carcinoma beyond the Milan criteria: a retrospective analysis. Am J Cancer Res 2015;5:450-457.

6. Lee SY, Konstantinidis IT, Eaton AA, Gönen M, Kingham TP, D’Angelica MI, et al. Predicting recurrence patterns after resection of hepatocellular cancer. HPB (Oxford) 2014;16:943-953.

7. Ma KW, She WH, Cheung TT, Chan ACY, Dai WC, Fung JYY et al. Validated nomogram for the prediction of disease-free survival after hepatectomy for hepatocellular carcinoma within the Milan criteria: individualizing a surveillance strategy. Surgery Today. 2019; 49(6):521-528.

8. Shengtao Lin, Feng Ye, Weiqi Rong, Ying Song, Fan Wu, Yunhe Liu et al. Nomogram to Assist in Surgical Plan for Hepatocellular Carcinoma: a Prediction Model for Microvascular Invasion. Journal of Gastrointestinal Surgery. 2019(2),https://doi.org/10.1007/s11605-019-04140-0.

9. Feng K, Yan J, Li X, Xia F, Ma K, Wang S et al. A randomized controlled trial of radiofrequency ablation and surgical resection in the treatment of small hepatocellular carcinoma. J Hepatol 2012;57:794-802.

10. Tao Lv, Li Jiang, Lunan Yan, Jiayin Yang, Bo Li, Tianfu Wen et al. Multiple Tumors Located in the Same Section Are Associated with Better Outcomes After Hepatic Resection for HCC Patients Meeting the Milan Criteria. J Gastrointest Surg. 2015; 19:2207-2214. 
11. Wei Zhang, Li Jiang, Lunan Yan, Jiayin Yang, Bo Li, Tianfu Wen et al. Radiofrequency ablation for HCC patients with multifocal tumours meeting the Milan criteria: A single-centre experience. Digestive and Liver Disease. 2016;48(12):1485-1491.

12. Francis Y. Yao, Linda Ferrell, Nathan M. Bass, Jessica J. Watson, Peter Bacchetti, Alan Venook et al. Liver transplantation for hepatocellular carcinoma: expansion of the tumor size limits does not adversely impact survival. Hepatology. 2001;33:1394-1403.

13. Hasegawa K, Kokudo N, Imamura H, Matsuyama Y, Aoki T, Minagawa M, et al. Prognostic impact of anatomic resection for hepatocellular carcinoma. Annals of surgery. 2005; 242(2):252-9.

14. Regimbeau J-M, Kianmanesh R, Farges O, Dondero F, Sauvanet A, Belghiti J. Extent of liver resection influences the outcome in patients with cirrhosis and small hepatocellular carcinoma. Surgery. 2002;131(3):311-7.

15. Fan ST, Mau Lo C, Poon RT, Yeung C, Leung Liu C, Yuen WK, et al. Continuous improvement of survival outcomes of resection of hepatocellular carcinoma: a 20-year experience. Ann Surg. 2011;253(4):745-58.

16. K. Bholee, K. Peng, Z. Zhou, J. Chen, L. Xu, Y. Zhang et al. Radiofrequency ablation combined with transarterial chemoembolization versus hepatectomy for patients with hepatocellular carcinoma within Milan criteria: a retrospective case-control study. Clin Transl Oncol. 2017; 19(7):844-852.

17. Ka Wing Ma, Wong Hoi She, Tan To Cheung, Albert Chi Yan Chan, Wing Chiu Dai, James Yan Yue Fung et al.Validated nomogram for the prediction of disease-free survival after hepatectomy for hepatocellular carcinoma within the Milan criteria: individualizing a surveillance strategy. Surgery Today. 2019;49(6):521-528.

18. Satoru Imura, Hiroki Teraoku, Masato Yoshikawa, Daichi Ishikawa, Shinichiro Yanada, Yu Saito et al.Potential predictive factors for microvascular invasion in hepatocellular carcinoma classifed within the Milan criteria.Int Clin Oncol. 2018; 23(1):98-103.

19. Eisuke Adachi, Shin-ichiro Maehara, Eiji Tsujita, Ken-ichi Taguchi, Shin-ichi Aishima, Tatsuya Rikimaru et al. Clinicopathologic risk factors for recurrence after a curative hepatic resection for hepatocellular carcinoma. Surgery 2002;131:S148-S152.

20. Ronnie Poon, Sheung-Tat Fan, Chung-Mau Lo, Chi-Leung Liu, John Wong. Intrahepatic recurrence after curative resection of hepatocellular carcinoma: long-term results of treatment and prognostic factors. Ann Surg. 1999; 229:216-222.

21. Shimada K, Sano T, Sakamoto Y, Kosuge T et al. A long-term followup and management study of hepatocellular carcinoma patients surviving for 10 years or longer after curative hepatectomy. Cancer (Phila); 2005; 104:1939-1947.

22. Hasegawa K, Kokudo N, Imamura H, Matsuyama Y, Aoki T, Minagawa M et al. Prognostic impact of anatomic resection for hepatocellular carcinoma. Annals of surgery. 2005; 242(2):252-9.

23. Eguchi S, Kanematsu T, Arii S, Okazaki M, Okita K, Omata M, et al. Comparison of the outcomes between an anatomical subsegmentectomy and a non-anatomical minor hepatectomy for single hepatocellular carcinomas based on a Japanese nationwide survey. Surgery. 2008; 143(4):469-75. 
24. Annette S. H. Gouw, Charles Balabaud, Hironori Kusano, Satoru Todo, Takafumi Ichida, and Masamichi Kojiro. Markers for Microvascular Invasion in Hepatocellular Carcinoma: Where Do We Stand? Liver Transplantation. 2011; 2:S72-80.

25. Timothy M. Pawlik, Keith A. Delman, Jean-Nicolas Vauthey, David M. Nagorney, Irene Oi-Lin Ng, Iwao Ikai et al. Tumor Size Predicts Vascular Invasion and Histologic Grade: Implications for Selection of Surgical Treatment for Hepatocellular Carcinoma. Liver Transplantation.2005; 11(9):1086-92.

26. Yan-Yan Wang, Jian-Hong Zhong, Hai-Feng Xu, Gang Xu, Li-Jun Wang, Da Xu et al. A modified staging of early and intermediate hepatocellular carcinoma based on single tumour $>7 \mathrm{~cm}$ and multiple tumours beyond up-to-seven criteria. Aliment Pharmacol Ther. 2018;1-9.

27. McHugh PP, Gilbert J, Vera S, Koch A, Ranjan D, Gedaly R, et al. Alpha-fetoprotein and tumour size are associated with microvascular invasion in explanted livers of patients undergoing transplantation with hepatocellular carcinoma. HPB (Oxford). 2010;12(1):56-61.

28. Sakata J, Shirai Y, Wakai T, Kaneko K, Nagahashi M, Hatakeyama K, et al. Preoperative predictors of vascular invasion in hepatocellular carcinoma. Eur J Surg Oncol. 2008; 34: 900-5.

29. Shao-liang Zhu, Jian-Hong Zhong, Yang ke, Liang Ma, Xue-Mei You, Le-Qun Li, et al. Efficacy of hepatic resection vs transarterial chemoembolization for solitary huge hepatocellular carcinoma. World J Gastroenterol 2015 August 28; 21(32): 9630-9637.

30. Jinli Zheng, Shu shen, Li jiang, Lunan Yan, Jiayin Yang, Bo Li, et al. Outcomes of anterior approach major hepatectomy with diaphragmatic resection for single huge right lobe HCC with diaphragmatic invasion.Medicine.2018;97:36.

31. The Cancer of the Liver Italian Program (CLIP) investigators. A new prognostic system for hepatocellular carcinoma: a retrospective study of 435 patients. Hepatology 1998;28:751-755.

32. Okuda K, Ohtsuki T, Obata $\mathrm{H}$, et al. Natural history of hepatocellular carcinoma and prognosis in relation to treatment. Study of 850 patients. Cancer 1985;56:918-928.

33. Tanizaki H, Ryu M, Kinoshita T, et al. Comparison of clinical features and survival in patients with hepatitis B and C virusrelated hepatocellular carcinoma. Jpn J Clin Oncol 1997;27:67-70.

34. Soo-Ho Lee, Jun-Suh Lee, Gun-Hyung Na, Young-Kyoung You, Dong-Goo Kim. Immunohistochemical markers for hepatocellular carcinoma prognosis after liver resection and liver transplantation. Clin Transplant. 2017; 31(1) doi: 10.1111/ctr.12852.

\section{Figures}




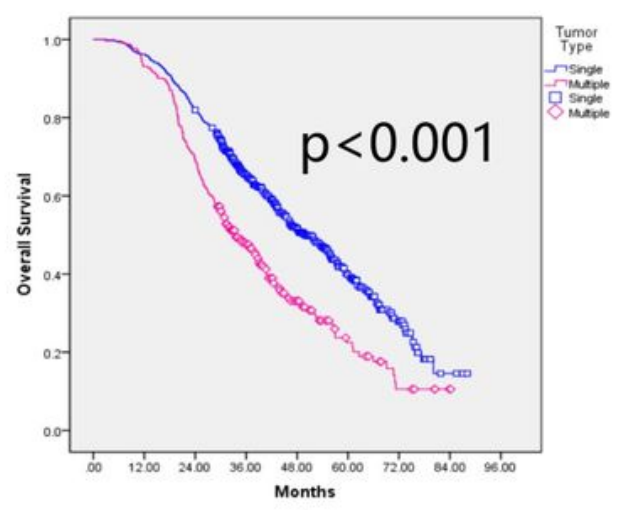

A

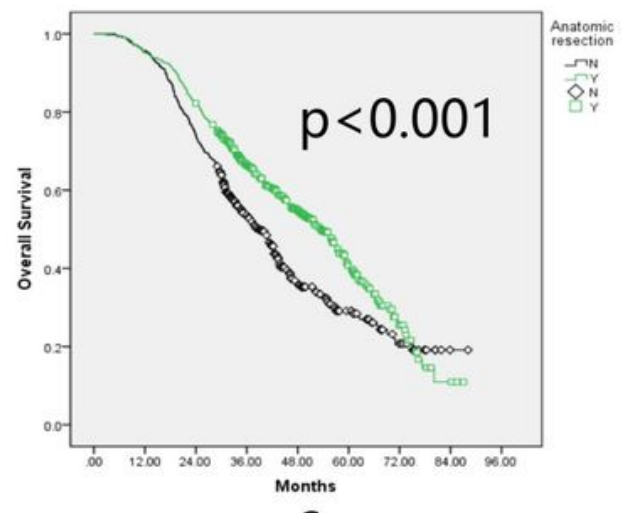

C

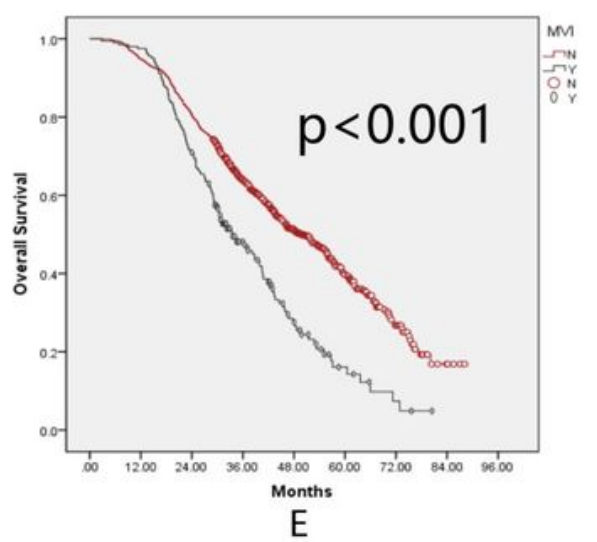

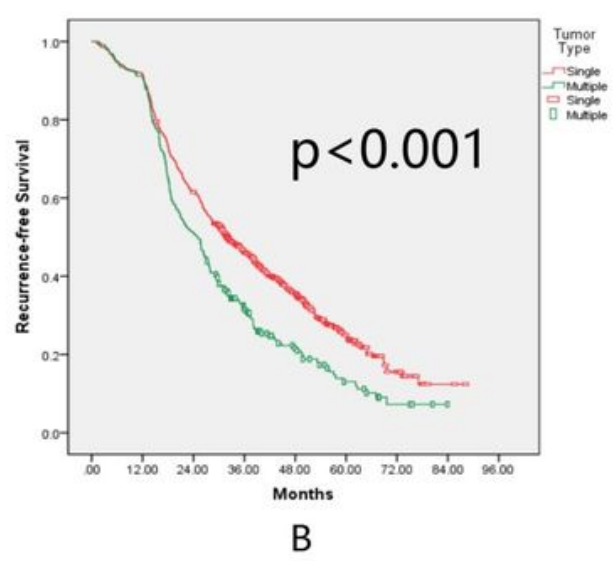
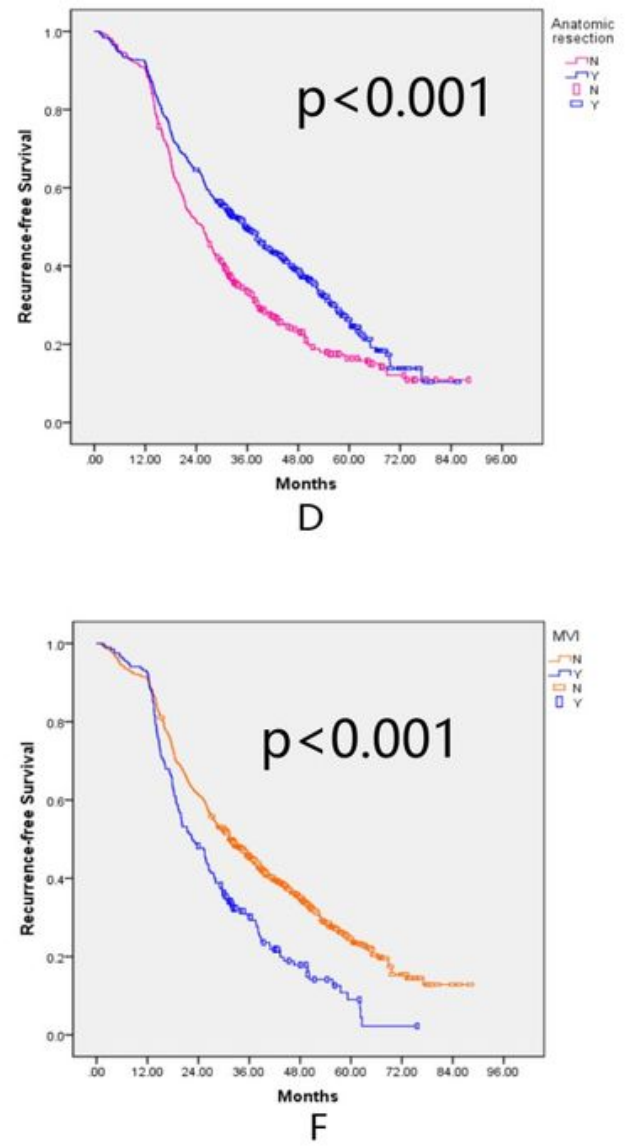

\section{Figure 1}

The OS and RFS for patients who were enrolled in this study. A. The 1-, 3- and 5-year OS for patients with sing and multiple tumor $(96.2 \%, 65.3 \%$ and $40.1 \%$ versus $93.1 \%, 47.6 \%$ and $22.5 \%$,). B. The $1-, 3$ - and $5-$ year RFS for patients with sing and multiple tumor $(91.4 \%, 46.2 \%$, and $24.6 \%$ versus $90.8 \%, 31.1 \%$ and $13.0 \%)$. C. The 1-, 3- and 5-year OS for patients in the anatomic resection and local resection $(95.2 \%$, $66.4 \%$ and $40.7 \%$ versus $95.8 \%, 53.6 \%$ and $29.1 \%$ ). D. The $1-, 3$ - and 5 -year RFS for patients in the 
anatomic resection and local resection ( $91.9 \%, 49.6 \%$ and $25.8 \%$ versus $90.4 \%, 33.5 \%$ and $16.4 \%)$. E. The 1-, 3- and 5-year OS for patients with MVI-positive and MVI-negative $(97.5 \%, 48.1 \%$ and $16.1 \%$ versus $94.9 \%, 63.8 \%$ and $39.7 \%)$. F. The 1-, 3- and 5-year OS for patients with MVI-positive and MVI-negative $(92.6 \%, 30.1 \%$ and $9.0 \%$ versus $90.9 \%, 45.5 \%$ and $24.4 \%)$.
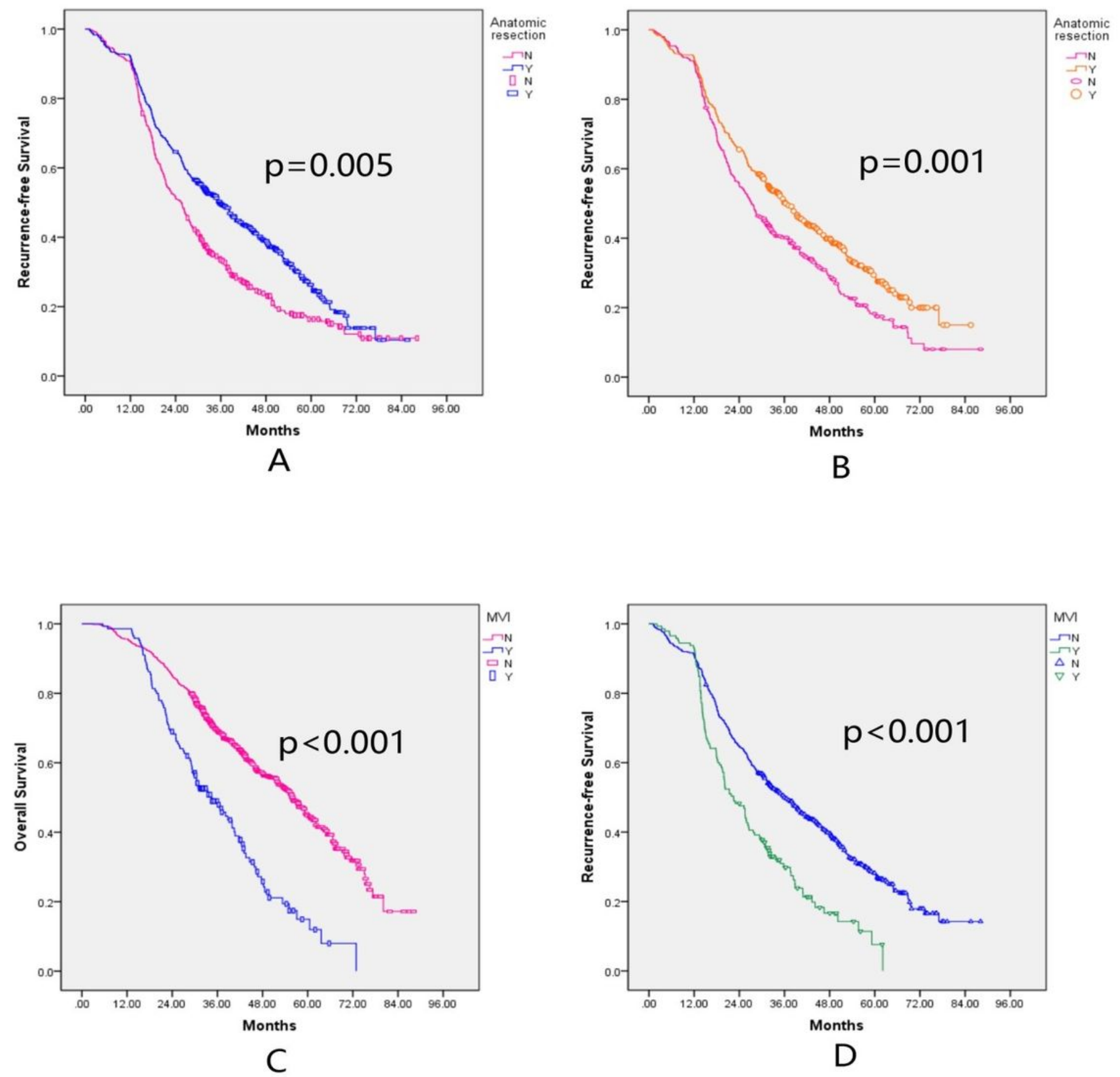

\section{Figure 2}

The OS and RFS for the patients with single tumor. A. The 1-, 3- and 5-year OS for patients in the anatomic resection and local resection $(95.7 \%, 69.2 \%$ and $42.9 \%$ versus $97.0 \%, 59.6 \%$ and $35 \%)$. B. The 
1-, 3- and 5-year RFS for patients in the anatomic resection and local resection (91.8\%, 50.3\% and 28.8\% versus $90.8 \%, 40.2 \%$ and $18.3 \%$ ). C. The 1-, 3- and 5-year OS for patients with MVI-positive and MVInegative (95.6\%, 48.9\% and $17.4 \%$ versus $95.7 \%, 69.3 \%$ and $44.8 \%)$. D. The $1-, 3$ - and 5 -year RFS for patients with MVI-positive and MVI-negative (93.1\%, $29.8 \%$ and $11.4 \%$ versus $92.3 \%, 50.0 \%$ and $27.8 \%$ ).

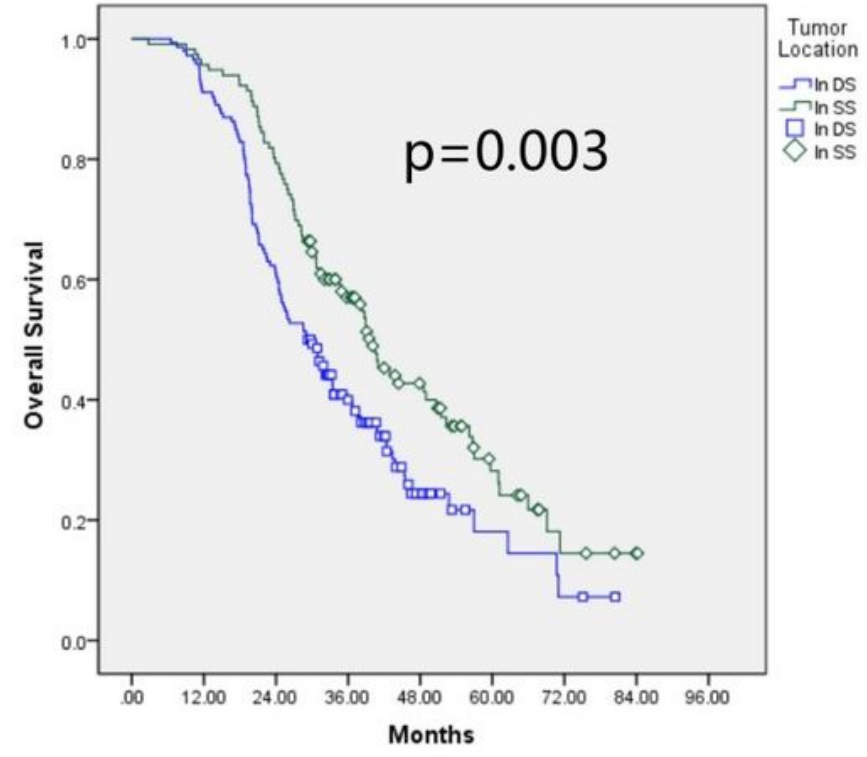

A

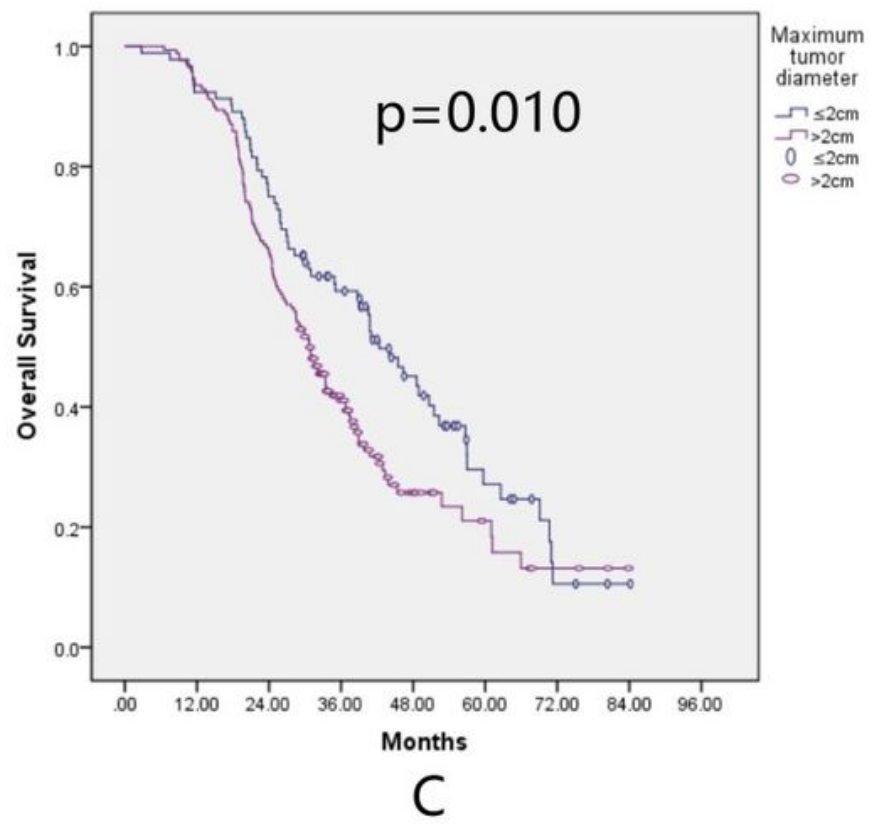

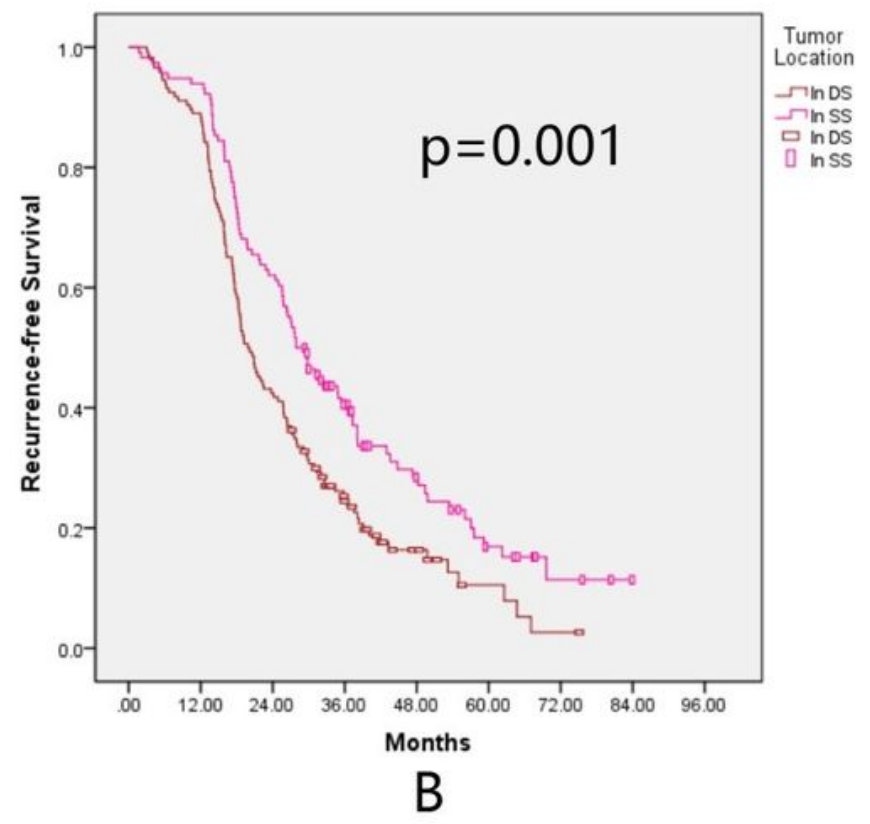

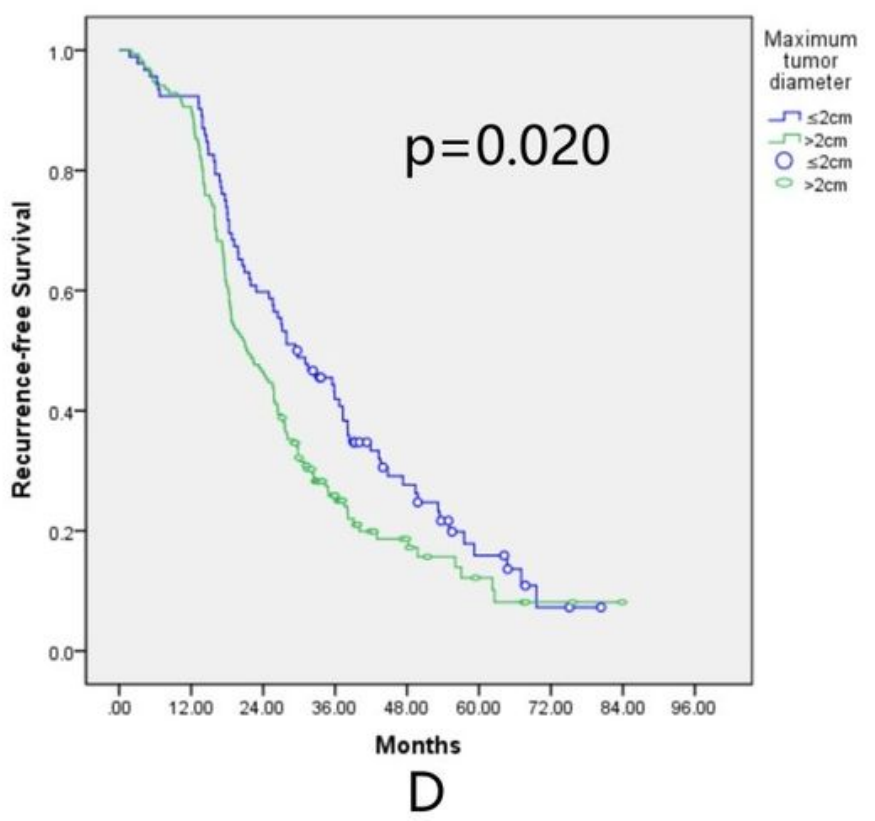

Figure 3 
The OS and RFS for the patients with multiple tumors. A. The 1- 3- and 5-year OS for patients with tumors located in different segments and same segment $(94.1 \%, 40.0 \%$ and $18.1 \%$ versus $95.7 \%, 57.0 \%$ and $28.2 \%)$. B. The 1-, 3- and 5-year RFS for patients with tumors located in different segments and same segment $(88.4 \%, 24.4 \%$ and $10.5 \%$ versus $94.0 \%, 40.5 \%$ and $16.9 \%$ ). C. The $1-, 3$ - and 5 -year OS for patients with maximum tumors diameter more than $2 \mathrm{~cm}(93.5 \%, 41.1 \%$ and $21.1 \%$ versus $92.1 \%, 59.3 \%$ and $27.2 \%)$. D. The 1-, 3- and 5-year OS for patients with maximum tumors diameter more than $2 \mathrm{~cm}$ $(90.0 \%, 25.9 \%$ and $12.2 \%$ versus $91.3 \%, 41.9 \%$ and $15.9 \%)$. 